\title{
A SYSTEM OF PDES FOR RIEMANNIAN SPACES
}

\author{
PADMA SENARATH, GILLIAN THORNLEY and BRUCE VAN BRUNT ${ }^{\curvearrowleft}$
}

(Received 31 March 2005; revised 22 January 2006)

Communicated by C. D. Hodgson

\begin{abstract}
Matsumoto [10] remarked that some locally projectively flat Finsler spaces of non-zero constant curvature may be Riemannian spaces of non-zero constant curvature. The Riemannian connection, however, must be metric compatible, and this requirement places restrictions on the geodesic coefficients for the Finsler space in the form of a system of partial differential equations. In this paper, we derive this system of equations for the case where the geodesic coefficients are quadratic in the tangent space variables $y^{i}$, and determine the solutions. We recover two standard Riemannian metrics of non-zero constant curvature from this class of solutions.
\end{abstract}

2000 Mathematics subject classification: primary 53B40; secondary $58 \mathrm{~J} 60$.

\section{Introduction}

This paper is concerned with conditions under which a locally projectively flat Finsler space of non-zero constant curvature is also a Riemannian space of non-zero constant curvature. The requirement that the Riemannian connection be metric compatible leads to a system of partial differential equations. We present this result in Section 2 along with a proof. In Section 3 we derive the solutions to the system of partial differential equations, and use them to obtain some Riemannian metrics for spaces of non-zero constant curvature. The remainder of this section is devoted to a review of certain basic definitions and results in Finsler geometry relevant to the study.

Let $F$ be a Finsler metric on an $n$-dimensional manifold $M$. The corresponding Finsler space is denoted by $\mathbf{F}^{n}$. Let $x=\left(x^{i}\right)$ denote the coordinates of $p \in M$, $y=\left(y^{i}\right) \in T_{p} M$, and

$$
g_{i j}=\frac{1}{2} \frac{\partial^{2} F^{2}}{\partial y^{i} \partial y^{j}} .
$$

(C) 2007 Australian Mathematical Society $1446-7887 / 07 \$ A 2.00+0.00$ 
The inverse of $\left(g_{i j}\right)$ is denoted by $\left(g^{i j}\right)$, that is, using the Einstein summation convention, $g^{j k} g_{i j}=\delta_{i}^{k}$, where

$$
\delta_{i}^{k}= \begin{cases}0 & \text { if } k \neq i, \\ 1 & \text { if } k=i\end{cases}
$$

is the Kronecker delta. The metric $F$ is called Riemannian if the $g_{j k}$ depend only on $x \in M$; it is called locally Minkowskian if the $g_{j k}$ depend only on $y \in T_{p} M$. For a non-zero vector $y \in T_{p} M$, and any vectors $u=u^{i} \partial / \partial x^{i}, v=v^{i} \partial / \partial x^{i} \in T_{p} M$, the inner product on $T_{p} M$ is given by $g_{y}(u, v)=g_{i j}(x, y) u^{i} v^{j}$. The definition of a Finsler metric implies that $g_{y}(y, y)=F^{2}$. Let $l_{i}=\partial F / \partial y^{i}$ and $h_{i j}(x, y)=g_{i j}-l_{i} l_{j}$. The angular form $h_{y}$ on $T_{p} M$ associated with the non-zero vector $y$ is defined by

$$
h_{y}(u, v)=h_{i j}(x, y) u^{i} v^{j}
$$

Let $h_{i}^{k}=g^{j k} h_{i j}$. The definitions of $g^{j k}$ and $h_{i j}$ give $h_{i}^{k}=\delta_{i}^{k}-l_{i} l^{k}$, where $l^{i}=y^{i} / F$.

The geodesics of $F$ are characterized locally by the condition

where

$$
\frac{d^{2} x^{i}}{d t^{2}}+2 G^{i}\left(x, \frac{d x}{d t}\right)=0
$$

$$
G^{i}(x, y)=\frac{1}{4} g^{i k}\left\{\left(\frac{\partial^{2} F^{2}}{\partial x^{j} \partial y^{k}}\right) y^{j}-\frac{\partial F^{2}}{\partial x^{k}}\right\} .
$$

The $G^{i}$ are positively homogeneous functions of degree two in $y$ and are called the geodesic coefficients of $F$. Let

$$
G_{j}^{i}=\frac{\partial G^{i}}{\partial y^{j}} \quad \text { and } \quad G_{j k}^{i}=\frac{\partial G_{j}^{i}}{\partial y^{k}} .
$$

The $G_{j k}^{i}$ are called the coefficients of the Berwald connection, which was introduced by Berwald in $1926([2,3])$. If $G_{j k}^{i}=G_{j k}^{i}(x)$, then $F$ is called a Berwald metric. Riemannian metrics are thus a special class of Berwald metrics. The classification of Berwald metrics was conducted by Szabo in 1981 (see [14]).

If $F$ is a Riemannian metric, then $g_{i j}=g_{i j}(x)$ so that

$$
G^{i}=\frac{1}{2} \Gamma_{j k}^{i} y^{j} y^{k} \quad \text { and } \quad G_{j k}^{i}=\Gamma_{j k}^{i} .
$$

Here, the $\Gamma_{j k}^{i}$ denote the Christoffel symbols of the Riemannian connection

$$
\Gamma_{j k}^{i}=\frac{1}{2} g^{r i}\left(\frac{\partial g_{r j}}{\partial x^{k}}+\frac{\partial g_{r k}}{\partial x^{j}}-\frac{\partial g_{j k}}{\partial x^{r}}\right)
$$


The Riemannian connection must be compatible with the metric, that is,

$$
\frac{\partial g_{i j}}{\partial x^{k}}-g_{r j} \Gamma_{i k}^{r}-g_{i r} \Gamma_{j k}^{r}=0
$$

Let

$$
R_{k}^{i}=2 \frac{\partial G^{i}}{\partial x^{k}}-y^{j} \frac{\partial^{2} G^{i}}{\partial x^{j} \partial y^{k}}+2 G^{j} \frac{\partial^{2} G^{i}}{\partial y^{j} \partial y^{k}}-\frac{\partial G^{i}}{\partial y^{j}} \frac{\partial G^{j}}{\partial y^{k}} .
$$

The Riemann curvature $R_{y}$ is a family of linear maps on tangent spaces defined by

$$
R_{y}=\left.R_{k}^{i} d x^{k} \frac{\partial}{\partial x^{i}}\right|_{p}: T_{p} M \rightarrow T_{p} M
$$

For a tangent plane $\Pi=\operatorname{span}\{y, u\} \subset T_{p} M$, let

$$
K(\Pi, y)=\frac{g_{y}\left(R_{y}(u), u\right)}{g_{y}(y, y) g_{y}(u, u)-g_{y}(y, u) g_{y}(y, u)} .
$$

The quantity $K(\Pi, y)$ is called the flag curvature of $\{\Pi, y\}$. If $F$ is a Riemannian metric, then $K$ is independent of $y \in \Pi$ and corresponds to the sectional curvature of tangent planes $\Pi \in T_{p} M$. The flag curvature in Finsler geometry is thus an extension of the sectional curvature in Riemannian geometry (see [3] and [4] for more details).

If, for every fixed point $y \in T_{p} M$, the flag curvature is independent of the tangent plane $\Pi=\operatorname{span}\{y, u\} \subset T_{p} M$, then $F$ is said to be of scalar curvature at the point $p$. If $F$ has scalar curvature at all points in $M$, then $\mathbf{F}^{n}$ is called a Finsler space of scalar curvature. In this case, $K$ is independent of the plane, but it depends on the point, where the flagpole starts; thus, $K=K(x, y)$, and the scalar curvature of $F$ is $R_{k}^{i}=K F^{2} h_{k}^{i}$.

We summarize some well-known formulæ for a Finsler space $\mathbf{F}^{n}$. The proofs of these formulæ along with further comments can be found in [10]. We consider first the case when $n>2$. It can be shown that

$$
R_{j k}^{i}=K_{j} h_{k}^{i}-K_{k} h_{j}^{i}=\frac{\partial G_{j}^{i}}{\partial x^{k}}-\frac{\partial G_{k}^{i}}{\partial x^{j}}-G_{r j}^{i} G_{k}^{r}+G_{r k}^{i} G_{j}^{r},
$$

where

$$
K_{i}=\frac{F^{2}}{3} \frac{\partial K}{\partial y^{i}}+K y_{i}
$$

Note that $R_{j k}^{i} y^{j}=R_{k}^{i}$ and $y_{i}=g_{i j} y^{j}$. Let $H_{i j k}^{r}=\partial R_{j k}^{r} / \partial y^{i}, H_{i j}=H_{i j r}^{r}$, and

$$
H_{i}=\frac{\left(n H_{j i}+H_{i j}\right) y^{j}}{n-1} \text {. }
$$


The following relations can be established:

$$
\begin{aligned}
K_{i} y^{i} & =K F^{2}, \\
H_{i j} & =(n-2) \frac{\partial K_{j}}{\partial y^{i}}+\frac{3 K_{i} l_{j}}{F}+K\left(h_{i j}-2 l_{i} l_{j}\right), \\
H_{i} & =(n+1) K_{i} .
\end{aligned}
$$

We now consider the case when $n=2$. It is known that every two-dimensional Finsler space is of scalar curvature. The scalar curvature of $\mathbf{F}^{2}$ can be expressed succinctly in terms of the orthonormal Berwald frame $\left(l^{i}, m^{i}\right)$, namely,

$$
R_{j k}^{i}=F K m^{i}\left(l_{j} m_{k}-m_{j} l_{k}\right),
$$

where $m_{i}=g_{i j} m^{j}$. In this case $K$ is called the Gauss curvature.

A Finsler space is said to be of constant flag curvature if there is a constant $\lambda$ such that $K(\Pi, y)=\lambda$ for all $y \in \Pi \subset T_{p} M, p \in M$.

Let $F$ and $\bar{F}$ be Finsler metrics on the $n$-dimensional manifold $M$, and let $\mathbf{F}^{n}=$ $(M, F)$ and $\overline{\mathbf{F}}^{n}=(M, \bar{F})$ be the corresponding Finsler spaces. A projective change is a difffeomorphism from $\mathbf{F}^{n}$ to $\overline{\mathbf{F}}^{n}$ that maps geodesics of $\mathbf{F}^{n}$ to geodesics of $\overline{\mathbf{F}}^{n}$. If any geodesic of $\mathbf{F}^{n}$ is a geodesic of $\overline{\mathbf{F}}^{n}$ and the converse is also true, then the change $F \rightarrow \bar{F}$ of the metric is called a projective change, and $\mathbf{F}^{n}$ is said to be projective to $\overline{\mathbf{F}}^{n}$. A Finsler metric $F$ is said to be locally projectively flat if there exists a projective change $F \rightarrow \bar{F}$ such that $\overline{\mathbf{F}}^{n}$ is a locally Minkowski space.

THEOREM 1.1 ([11]). The change $F \rightarrow \bar{F}$ of the metric is projective if and only if

$$
\bar{F}_{\mathrm{j}}-\frac{\partial \bar{F}_{\mid k}}{\partial y^{i}} y^{k}=0,
$$

where $\bar{F}_{\mid k}$ denotes the horizontal covariant derivative of $\bar{F}$ on $\mathbf{F}^{n}$, that is,

$$
\bar{F}_{\mid k}=\frac{\partial \bar{F}}{\partial x^{k}}-\frac{\partial G^{r}}{\partial y^{k}} \frac{\partial \bar{F}}{\partial y^{r}} .
$$

Here, the $G^{r}$ denote the geodesic coefficients of $F$. We say that $\mathbf{F}^{n}$ is projective to $\overline{\mathbf{F}}^{n}$ with the projective factor given by $P=\bar{F}_{\mid k} y^{k} / 2 \vec{F}$.

THEOREM 1.2 ([1]). A Finsler space $\mathbf{F}^{n}=(M, F)$ is a projectively flat Berwald space if and only if it belongs to one of the following classes:

(a) locally Minkowski spaces;

(b) Riemannian spaces of constant curvature;

(c) spaces $\mathbf{F}^{2}$ with a metric of the form $F=\beta^{2} / \gamma$, where $\beta$ and $\gamma$ are one-forms. 
LEMMA 1.3 ([14]). Let $M$ be a two-dimensional manifold and $F$ be a positive definite Berwald metric on $M$. Then $F$ is either locally Minkowskian or Riemannian.

THEOREM 1.4 ([9]). A Finsler space of scalar curvature is a space of constant curvature if and only if one of the following conditions hold:

(i) $H_{i j}=H_{j i}$;

(ii) $H_{j i} y^{j}=H_{i j} y^{j}$;

(iii) $H_{i}$ is proportional to $y_{i}$.

\section{Properties of projectively flat Finsler spaces}

This section is devoted to a proof of the following result.

THEOREM 2.1. Let $\mathbf{F}^{n}=(M, F)$ be a Finsler space that has geodesic coefficients of the form $G^{k}=\rho_{r} y^{r} y^{k}$ with $\rho_{i} \rho_{j}-\rho_{i j}$ non-degenerate, where $\rho$ is a smooth scalar function on $M$ and $\rho_{r}=\partial \rho / \partial x^{r}$. Then

(a) $\mathbf{F}^{n}$ is locally projectively flat;

(b) $\mathbf{F}^{n}$ is of constant curvature;

(c) $\mathbf{F}^{n}$ is a Riemannian space of non-zero constant sectional curvature given by

$$
K=\frac{1}{F^{2}}\left(\rho_{i} \rho_{j}-\rho_{i j}\right) y^{i} y^{j}
$$

(d) $\rho$ satisfies the the system of partial differential equations

$$
\rho_{i j k}-2\left(\rho_{i j} \rho_{k}+\rho_{j k} \rho_{i}+\rho_{i k} \rho_{j}\right)+4 \rho_{i} \rho_{j} \rho_{k}=0,
$$

where $\rho_{i j}=\partial \rho_{i} / \partial x^{j}$ and $\rho_{i j k}=\partial \rho_{i j} / \partial x^{k}$.

Proof. (a) We use Theorem 1.1 to show that $\mathbf{F}^{n}$ is locally projectively flat. Let $\overline{\mathbf{F}}^{n}=(M, \bar{F})$ be a locally Minkowski space. It is thus required to show that equation (1.8) is satisfied. Since $\bar{F}$ does not depend on $x, \bar{F}_{\mid i}=-G_{i}^{k} \partial \bar{F} / \partial y^{k}$, and equation (1.8) can be written

$$
G_{i}^{k} \frac{\partial \bar{F}}{\partial y^{k}}+\frac{\partial \bar{F}_{\mid k}}{\partial y^{i}} y^{k}=0 .
$$

Now, $G^{k}=\rho_{r} y^{r} y^{k}$, and differentiating with respect to $y^{i}$ gives

$$
G_{i}^{k}=\rho_{i} y^{k}+\rho_{r} y^{r} \delta_{i}^{k}
$$


hence,

$$
\bar{F}_{\mid i}=-\rho_{i} y^{k} \frac{\partial \bar{F}}{\partial y^{k}}-\rho_{r} y^{r} \frac{\partial \bar{F}}{\partial y^{i}}=-\rho_{i} \bar{F}-\rho_{r} y^{r} \frac{\partial \bar{F}}{\partial y^{i}}
$$

where we have used the homogeneity property of $\bar{F}$. Differentiating this equation with respect to $y^{i}$ and contracting with $y^{k}$ yields

$$
\frac{\partial \bar{F}_{\mid k}}{\partial y^{i}} y^{k}=-\rho_{k} \frac{\partial \bar{F}}{\partial y^{i}} y^{k}-\rho_{i} \frac{\partial \bar{F}}{\partial y^{k}} y^{k}-\rho_{r} y^{r} \frac{\partial^{2} \bar{F}}{\partial y^{i} \partial y^{k}} y^{k},
$$

and the homogeneity of $\vec{F}$ gives

$$
\frac{\partial \bar{F}_{\mid i}}{\partial y^{i}} y^{k}=-\rho_{k} \frac{\partial \bar{F}}{\partial y^{i}} y^{k}-\rho_{i} \bar{F}
$$

Equations (2.4) and (2.6) imply that equation (2.3) is satisfied and hence, by Theorem $1.1, \mathbf{F}^{n}$ is locally projectively flat.

(b) We show here that $\mathbf{F}^{n}$ is of constant curvature for the case $n>2$. The case $n=2$ is discussed in the proof of part (c). It is known that locally projectively flat Finsler metrics are of scalar curvature (see [6] and [5]). Part (a) thus shows that $\mathbf{F}^{n}$ is of scalar curvature. To establish that $\mathbf{F}^{n}$ is of constant curvature it is thus sufficient to show that $H_{i j}=H_{j i}$.

Now, $H_{i j k}^{r}=\partial R_{j k}^{r} / \partial y^{i}$, where $R_{j k}^{r}$ is given by (1.3), and equation (2.4) can be used to calculate $G_{j m}^{r}$. Differentiation of equation (2.4) with respect to $y^{m}$ and change of indices leads to

$$
G_{j m}^{r}=\rho_{j} \delta_{m}^{r}+\rho_{m} \delta_{j}^{r}
$$

Consequently (1.3) becomes

$$
R_{j k}^{r}=\left(\rho_{i k} \delta_{j}^{r}-\rho_{i j} \delta_{k}^{r}\right) y^{i}+\left(\rho_{j} \delta_{k}^{r}-\rho_{k} \delta_{j}^{r}\right) \rho_{i} y^{i}
$$

where we have used $\rho_{i j}=\rho_{j i}$. Differentiating equation (2.8) with respect to $y^{i}$ gives

$$
\frac{\partial R_{j k}^{r}}{\partial y^{i}}=H_{i j k}^{r}=\rho_{i k} \delta_{j}^{r}-\rho_{i j} \delta_{k}^{r}+\left(\rho_{j} \delta_{k}^{r}-\rho_{k} \delta_{j}^{r}\right) \rho_{i},
$$

which, using $k=r$ and noting that $\delta_{r}^{r}=n$ and $H_{i j}=H_{i j r}^{r}$, yields

$$
H_{i j}=(n-1)\left(\rho_{i} \rho_{j}-\rho_{i j}\right)=H_{j i}
$$

Theorem (1.4) thus shows that $\mathbf{F}^{n}$ is of constant curvature. 
(c) To establish equation (2.1) we consider two cases.

Case (i) $n>2$. Equations (1.4) and (2.9) imply $H_{i}=(n+1)\left(\rho_{i} \rho_{j}-\rho_{i j}\right) y^{j}$, and equation (1.6) thus shows that $K_{i}=\left(\rho_{i} \rho_{j}-\rho_{i j}\right) y^{j}$. Contracting the above expression with $y^{i}$ and using equation (1.5) gives equation (2.1). Since $\rho_{i} \rho_{j}-\rho_{i j}$ is non-degenerate, $K \neq 0$, and

$$
F^{2}=\frac{1}{K}\left(\rho_{i} \rho_{j}-\rho_{i j}\right) y^{i} y^{j}=g_{i j}(x) y^{i} y^{j} ;
$$

hence, the metric tensor is given by

$$
g_{i j}=\frac{1}{K}\left(\rho_{i} \rho_{j}-\rho_{i j}\right)
$$

Since the metric tensor depends only on $x, F$ is Riemannian and hence $F^{n}$ is a Riemannian space of non-zero sectional curvature given by equation (2.1).

Case (ii) $n=2$. It is known that any two-dimensional Finsler space is of scalar curvature. Contracting equation (1.7) with $m_{i}, l^{j}$ and $m^{k}$ gives $K F=R_{j k}^{i} m_{i} l^{j} m^{k}$, and equation (2.8) yields

$$
K F=\left\{\left(\rho_{r k} \delta_{j}^{i}-\rho_{r j} \delta_{k}^{i}\right) y^{r}+\left(\rho_{j} \delta_{k}^{i}-\rho_{k} \delta_{j}^{i}\right) \rho_{r} y^{r}\right\} m_{i} l^{j} m^{k}=-\rho_{r j} l^{j} y^{r}+\rho_{j} l^{j} \rho_{r} y^{r} .
$$

Now, $l^{i}=y^{i} / F$ and hence $K=\left(\rho_{i} \rho_{j}-\rho_{i j}\right) y^{i} y^{j} / F^{2}$, where $i, j=1,2$.

We show now that $\mathbf{F}^{2}$ is a Riemannian space of non-zero constant sectional curvature. Since $F$ is a Berwald metric, Lemma 1.3 shows that $F$ is either locally Minkowskian or Riemannian. Recall that if $F$ is locally Minkowskian then $G^{i}=0$, which contradicts a hypothesis of the theorem; hence, $F$ is Riemannian. Part (a) shows that $F$ is a locally projectively flat Berwald metric. Theorem 1.2 thus indicates that $F$ is a Riemannian metric of non-zero constant sectional curvature.

(d) Since $\mathbf{F}^{n}$ is a Riemannian space, the metric must be compatible with the Riemannian connection. In other words, the metric must satisfy equation (1.2) and the coefficients of the Berwald connection coincide with the Christoffel symbols of the Riemannian connection. Equation (2.7) thus implies $\Gamma_{j k}^{i}=G_{j k}^{i}=\rho_{k} \delta_{j}^{i}+\rho_{j} \delta_{k}^{i}$. Equation (1.2) can thus be written

$$
\frac{\partial g_{i j}}{\partial x^{k}}=g_{r j}\left(\rho_{i} \delta_{k}^{r}+\rho_{k} \delta_{i}^{r}\right)+g_{i r}\left(\rho_{k} \delta_{j}^{r}+\rho_{j} \delta_{k}^{r}\right)=2 g_{i j} \rho_{k}+g_{k j} \rho_{i}+g_{i k} \rho_{j} .
$$

Equation (2.10) can be used to write the above equation in terms of the derivatives of $\rho$. In particular, $K$ is constant and therefore differentiating equation (2.10) with respect to $x^{k}$ gives

$$
\frac{\partial g_{i j}}{\partial x^{k}}=\frac{1}{K}\left(\rho_{i k} \rho_{j}+\rho_{j k} \rho_{i}-\rho_{i j k}\right)
$$

moreover, equation (2.10) can be used directly to eliminate the metric tensor components from equation (2.11). This elimination leads to equation (2.2). 
A partial converse to the above theorem is available in the following form.

THEOREM 2.2. Let $\mathbf{F}^{n}=(M, F)$ be a Riemannian space defined on an underlying manifold of dimension $n$, and let

$$
F^{2}=\mu\left(\rho_{i} \rho_{j}-\rho_{i j}\right) y^{i} y^{j}
$$

with $\rho_{i} \rho_{j}-\rho_{i j}$ non-degenerate, where $\mu$ is a non-zero constant, and $\rho_{i}=\rho_{i}(x)$ is a scalar field defined on $M$ that satisfies equation (2.2). Then

$$
G^{i}=\rho_{r} y^{r} y^{i}
$$

and the curvature of $\mathbf{F}^{n}$ is $1 / \mu$.

PROOF. Differentiating equation (2.12) and substituting into (1.1) leads to

$$
G^{i}=\frac{1}{4} \mu g^{i k}\left(2 \rho_{k} \rho_{j r}-\rho_{r k j}\right) y^{j} y^{r} .
$$

The functions $\rho_{r k j}$ can be eliminated from this expression using equation (2.2). Thus,

$$
G^{i}=\mu g^{i k}\left(\rho_{r} \rho_{k} \rho_{j}-\rho_{j k} \rho_{r}\right) y^{j} y^{r} .
$$

We know from the second derivative of (2.12) that the metric tensor of $\mathbf{F}^{n}$ is

$$
g_{k r}=\mu\left(\rho_{k} \rho_{r}-\rho_{k r}\right)
$$

Contracting this expression with $g^{i k}$ and substituting in (2.14) gives $G^{i}=\rho_{r} y^{r} y^{i}$. The conditions of Theorem 2.1 are now satisfied, and from equations (2.1) and (2.12) we have $K=1 / \mu$.

\section{Solutions to the system of partial differential equations}

Equation (2.2) forms an overdetermined system of partial differential equations for the function $\rho$. Typically, if such systems have solutions, then these solutions depend on arbitrary constants rather than arbitrary functions (see [13]). In this section we present a general family of solutions to system (2.2) and then specialize to radially symmetric solutions.

For the special choice $i=k=j$, the partial differential equation is

$$
\rho_{i i i}-6 \rho_{i i} \rho_{i}+4 \rho_{i}^{3}=0 .
$$


For fixed values of $x^{m}, m \neq i$, the above equation can be regarded as an ordinary differential equation. This observation leads to the study of the second order nonlinear ordinary differential equation

$$
u^{\prime \prime}(\xi)-6 u^{\prime}(\xi) u(\xi)+4 u^{3}(\xi)=0 .
$$

A solution $u=u\left(\xi, c_{1}, c_{2}\right)$ to equation (3.2) depends on two integration constants, say $c_{1}$ and $c_{2}$. A solution to the partial differential equation (3.1) can then be obtained by integration, namely,

$$
\rho(x)=\int_{x_{0}^{i}}^{x^{i}} u\left(\xi, c_{1}, c_{2}\right) d \xi+k .
$$

Here, $k$ is a constant, and the $c_{i}$ can be regarded as functions that depend on $x^{m}, m \neq i$. The functional dependence of the $c_{i}$ on the $x^{m}$ is constrained by the other equations in system (2.2).

Let $\xi_{0}, u_{0}$ and $u_{0}^{\prime}$ be given real numbers. We can exploit the theory of ordinary differential equations to glean qualitative information about the initial-value problem, which consists of finding solutions to equation (3.2) such that

$$
u\left(\xi_{0}\right)=u_{0}, \quad \text { and } \quad u^{\prime}\left(\xi_{0}\right)=u_{0}^{\prime} .
$$

Specifically, the Picard-Lindelöf Theorem (see [8]) can be invoked to show that there is a local solution to the initial-value problem and that the solution is unique among functions that are twice continuously differentiable. In fact, the solution can be found explicitly.

LEMMA 3.1. For any choice of initial values $\xi_{0}, u_{0}$ and $u_{0}^{\prime}$ there exists a local solution to equation (3.2) that satisfies equations (3.4). This solution is unique among functions that have continuous second derivatives in a neighbourhood of $\xi_{0}$.

(a) If $2 u_{0}^{2}-u_{0}^{\prime} \neq 0$, then

$$
u(\xi)=-\frac{\xi+c_{1}}{\left(\xi+c_{1}\right)^{2}+c_{2}},
$$

where $c_{1}=-\left(u_{0} /\left(2 u_{0}^{2}-u_{0}^{\prime}\right)+\xi_{0}\right)$ and $c_{2}=\left(u_{0}^{2}-u_{0}^{\prime}\right) /\left(2 u_{0}^{2}-u_{0}^{\prime}\right)^{2}$.

(b) If $2 u_{0}^{2}-u_{0}^{\prime}=0$ and $u_{0} \neq 0$, then

$$
u(\xi)=-\frac{1}{2\left(\xi+c_{1}\right)},
$$

where $c_{1}=-\left(1 /\left(2 u_{0}\right)+\xi_{0}\right)$. 
(c) If $2 u_{0}^{2}-u_{0}^{\prime}=0$ and $u_{0}=0$, then the local solution is the trivial solution $u(\xi)=0$.

Proof. The uniqueness of the solution to the initial-value problem follows from the Picard-Lindelöf Theorem for ordinary differential equations. In particular, the differential equation (3.2) can be recast as the system with $y_{1}=u$,

$$
\begin{aligned}
& y_{1}^{\prime}(\xi)=y_{2}(\xi)=f_{1}\left(\xi, y_{1}, y_{2}\right), \\
& y_{2}^{\prime}(\xi)=6 y_{1}(\xi) y_{2}(\xi)-4 y_{1}^{3}(\xi)=f_{2}\left(\xi, y_{1}, y_{2}\right) .
\end{aligned}
$$

The $f_{k}$ are analytic for all values of $\xi, y_{1}$ and $y_{2} ;$ a fortiori, these functions satisfy the requisite Lipschitz condition in a suitably small neighbourhood of the initial values for any choice of $\xi_{0}, u_{0}$ and $u_{0}^{\prime}$.

It can be verified by direct calculation that the functions defined by equations ( 3.5 ) and (3.6), along with the trivial solution, satisfy equation (3.2). It is straightforward algebra to show that the integration constants satisfy the initial conditions (3.4). The solution defined by equation (3.6) contains only a single integration constant owing to the condition $2 u_{0}^{2}-u_{0}^{\prime}=0$.

THEOREM 3.2. Let $\Omega \subseteq \mathbb{R}^{n}$ be a region and $\rho: \Omega \rightarrow \mathbb{R}$ be a function with continuous third-order derivatives. The function $\rho$ is a real solution to system (2.2) if and only if there exists a quadratic polynomial $Q: \mathbb{R}^{n} \rightarrow \mathbb{R}$ such that, for all $x \in \Omega$, $Q(x)>0$, and

$$
\rho(x)=-\frac{1}{2} \ln Q(x)
$$

ProOF. Suppose that $\rho$ is a function of the form defined by equation (3.7) and let $Q_{i}=\partial Q / \partial x^{i}, Q_{i j}=\partial Q_{i} / \partial x^{j}$ and $Q_{i j k}=\partial Q_{i j} / \partial x^{k}$. Then, for $i, j, k \in\{1, \ldots, n\}$,

$$
\begin{aligned}
\rho_{i} & =-\frac{1}{2} \frac{Q_{i}}{Q}, \quad \rho_{i j}=-\frac{1}{2}\left\{\frac{Q_{i j}}{Q}-\frac{Q_{i} Q_{j}}{Q^{2}}\right\}, \\
\rho_{i j k} & =\frac{1}{2 Q^{2}}\left\{Q_{i j} Q_{k}+Q_{i k} Q_{j}+Q_{j k} Q_{i}\right\}-\frac{Q_{i} Q_{j} Q_{k}}{Q^{3}}-\frac{Q_{i j k}}{2 Q} .
\end{aligned}
$$

These expressions for the derivatives of $\rho$ show that

$$
\rho_{i j k}-2\left(\rho_{i j} \rho_{k}+\rho_{i k} \rho_{j}+\rho_{j k} \rho_{i}\right)+4 \rho_{i} \rho_{j} \rho_{k}=-\frac{Q_{i j k}}{2 Q} .
$$

However, $Q$ is a quadratic polynomial and therefore $Q_{i j k}=0$ for all $i, j, k \in$ $\{1, \ldots, n\}$. Equation (2.2) is thus satisfied for all $i, j, k \in\{1, \ldots, n\}$. 
Suppose that $\rho$ is a solution to system (2.2). Then $\rho$ must satisfy equation (3.1) for $i=1, \ldots, n$. If $i=1$, then $\rho$ can be expressed in the form (3.3), where $c_{1}$ and $c_{2}$ are functions of $x^{2}, \ldots, x^{n}$. The uniqueness of the solutions to equation (3.2) shows that $u$ must be one of the solutions given in Lemma 3.1. Integrating any of the solution forms of Lemma 3.1 gives a solution of the form

$$
\rho(x)=-\frac{1}{2} \ln V(x),
$$

The calculation leading to equation (3.8) is still valid with $Q$ replaced by $V$, and since $\rho$ is a solution to system $(2.2)$ we have that $V_{i j k}=0$ for all $i, j, k \in\{1, \ldots, n\}$. Therefore, $V$ must be of the form $V(x)=A_{j k} x^{j} x^{k}+B_{j} x^{j}+C$, where $A_{j k}, B_{j}$ and $C$ are constants, that is, $V$ must be a quadratic polynomial.

Theorem 3.2 shows that any quadratic function $Q$ that is positive in a neighbourhood of a point $x_{0} \in \mathbb{R}^{n}$ leads to a real solution $\rho$ of the system (2.2). Not all such $Q$, however, generate a Riemannian metric and specifically a matrix $\left(g_{j k}\right)$ that is positive definite. We now consider the types of quadratic functions that lead to Riemannian metrics.

LEMMA 3.3. Suppose that $Q$ is a polynomial of degree $d \leq 2$ such that $Q\left(x_{0}\right)>0$ and the matrix $\left(g_{j k}\right)$ is positive definite in some neighbourhood $N\left(x_{0}\right)$ of $x_{0}$. Then there exists an affine transformation in $\mathbb{R}^{n}, x \rightarrow w$, such that $Q(x)=\hat{Q}(w)$ can be reduced to one of the following forms:

$$
\begin{aligned}
& \hat{Q}(w)=C+\left(w^{1}\right)^{2}+\cdots+\left(w^{p}\right)^{2}-\left(w^{p+1}\right)^{2}-\cdots-\left(w^{n}\right)^{2}, \\
& \hat{Q}(w)=C+w^{1}+\left(w^{2}\right)^{2}+\cdots+\left(w^{p+1}\right)^{2}-\left(w^{p+2}\right)^{2}-\cdots-\left(w^{n}\right)^{2},
\end{aligned}
$$

where $C$ is a constant. Here, $p$ denotes the number of quadratic terms with positive coefficients: $p=0$ corresponds to no quadratic terms with positive coefficients; $p=n$ corresponds to all quadratic terms having positive coefficients in (3.10); $p=n-1$ corresponds to all quadratic terms having positive coefficients in (3.11).

PROOF. The above result follows from a standard result about the canonical forms for quadratic functions (see [7], Chapter 11) once it is verified that $\hat{Q}$ must depend on all the $w^{k}$ and that $d=2$.

Since the matrix $\left(g_{j k}\right)$ is positive definite, the transformed matrix $\left(\hat{g}_{j k}\right)$ generated by $\hat{Q}$ is also positive definite. Equation (2.10) implies that

$$
\hat{g}_{j k}=\frac{1}{2 K \hat{Q}}\left(\hat{Q}_{j k}-\frac{1}{2} \frac{\hat{Q}_{j} \hat{Q}_{k}}{\hat{Q}}\right)
$$


Evidently, if $\hat{Q}$ is independent of $w^{j}$, then $\hat{g}_{j k}=0$ for all $k=1, \ldots n$; consequently, the corresponding matrix $\left(\hat{g}_{j k}\right)$ has a row of zeros and cannot be positive definite. We thus conclude that $\hat{Q}$ must depend on all of the $w^{j}$.

The Sylvester criteria for positive definiteness requires that

$$
\hat{g}_{11}=\frac{1}{2 K \hat{Q}}\left(\hat{Q}_{11}-\frac{1}{2} \frac{\hat{Q}_{1}^{2}}{\hat{Q}}\right)>0,
$$

and

$$
\hat{g}_{11} \hat{g}_{22}-\hat{g}_{12}^{2}=\frac{1}{4 K^{2} \hat{Q}^{2}}\left\{\hat{Q}_{11} \hat{Q}_{22}-\frac{1}{2 \hat{Q}}\left(\hat{Q}_{11} \hat{Q}_{2}^{2}+\hat{Q}_{22} \hat{Q}_{1}^{2}\right)\right\}>0
$$

(along with similar conditions for the other principal minors). Inequality (3.14) shows that $\hat{Q}$ cannot be a linear function. Hence, $\hat{Q}$ must contain some quadratic terms, so that $d=2$.

We now assume that $Q$ has been reduced to one of the forms given in Lemma 3.3 and that $Q(0)=C>0$. The circumflexes for the transformed quantities will be dropped and we use the variables $x^{k}$. The condition that the $\left(g_{j k}\right)$ matrix generated by $Q$ be positive definite further limits the possible forms for $Q$.

THEOREM 3.4. Suppose that the quadratic function $Q$ generates a Riemannian metric in a neighbourhood of $0 \in \mathbb{R}^{n}$ by use of equations (3.7) and (2.10).

(a) If $K<0$, then $Q$ is either of the form

$$
\begin{aligned}
& Q(x)=C-|x|^{2} \quad \text { or } \\
& Q(x)=C+x^{1}-\left(\left(x^{2}\right)^{2}+\cdots+\left(x^{n}\right)^{2}\right) .
\end{aligned}
$$

(b) If $K>0$, then $Q$ is of the form

$$
Q(x)=C+|x|^{2}
$$

Proof. (a) Let $K<0$. The quadratic function $Q$ is either of the form (3.10) or (3.11). It suffices to show that in either case $p=0$.

Suppose $Q$ is of the form (3.10) with $p>0$. Since the corresponding matrix $\left(g_{j k}\right)$ must be positive definite, (3.13) implies that $g_{11}=\left(Q-\left(x^{1}\right)^{2}\right) /\left(K Q^{2}\right)>0$; hence, $Q-\left(x^{1}\right)^{2}<0$. However, $Q(0)=c>0$, so that $Q-\left(x^{1}\right)^{2}>0$ for all $x^{i}$ sufficiently small. We thus conclude that $p=0$ and that $Q$ is of the form (3.15).

Suppose now that $Q$ is of the form (3.11) with $p>0$. Then $g_{11}=-1 /\left(4 K Q^{2}\right)>0$, so that inequality (3.13) is satisfied; however,

$$
g_{11} g_{22}-g_{12}^{2}=-\frac{1}{4 K^{2} Q^{3}}<0,
$$


so that inequality (3.14) is not satisfied. We thus have the contradiction that $\left(g_{j k}\right)$ is not positive definite; consequently, $p=0$ and $Q$ is of the form (3.16).

(b) Let $K>0$. If $Q$ is of the form (3.11) then $g_{11}=-1 /\left(4 K Q^{2}\right)<0$, and hence $\left(g_{j k}\right)$ is not positive definite. Therefore, $Q$ must be of the form (3.10). Suppose that $p<n$. Without loss of generality we can relabel variables so that

$$
Q(x)=C-\left(x^{1}\right)^{2}+q,
$$

where $q$ represents the remaining quadratic terms. Now, for $x^{i}$ sufficiently small, $g_{11}=-\left(Q+\left(x^{1}\right)^{2}\right) /\left(K Q^{2}\right)<0$, so that $\left(g_{j k}\right)$ is not positive definite. We thus have that $p=n$ and that $Q$ is of the form (3.17).

If $Q$ is of the form (3.15) or (3.17), then we recover known Riemannian metrics of constant curvature (see [12]). Now,

$$
F^{2}=g_{j k} y^{j} y^{k}=\frac{1}{2 K Q}\left(Q_{j k}-\frac{1}{2} \frac{Q_{j} Q_{k}}{Q}\right) y^{j} y^{k} .
$$

If $Q$ is of the form (3.15), then

$$
F^{2}=-\frac{1}{K Q}\left(|y|^{2}+\frac{\langle x, y\rangle^{2}}{Q}\right) .
$$

For the case $K=-1, C=1$, the above expression is

$$
F^{2}=\frac{|y|^{2}}{1-|x|^{2}}+\frac{\langle x, y\rangle^{2}}{\left(1-|x|^{2}\right)^{2}},
$$

which corresponds to the Klein metric on the unit ball. If $Q$ is of the form (3.16), then we discover a new Riemannian metric of the form

$$
\begin{aligned}
F^{2}= & -\frac{1}{K Q}\left(|y|^{2}+\frac{\langle x, y\rangle^{2}}{Q}\right) \\
& +\frac{1}{K Q}\left(\left(y^{1}\right)^{2}+\frac{y^{1}\left(1+2 x^{1}\right)\langle x, y\rangle^{2}}{Q}-\frac{\left(y^{1}\right)^{2}\left(1+2 x^{1}\right)^{2}}{4 Q}\right) .
\end{aligned}
$$

If $Q$ is of the form (3.17), then $F^{2}=\left(|y|^{2}-\langle x, y\rangle^{2} / Q\right) /(K Q)$, which is a Riemannian metric given in [12].

\section{Acknowledgement}

The authors wish to express their gratitude to Prof. Dr Z. Shen for sending valuable reference materials. 


\section{References}

[1] S. Bacso, 'Projective Finsler geometry', Paper presented at the workshop on Finsler Geometry, University of California, Berkeley, 2002.

[2] L. Berwald, 'Über Parallelubertragung in Räumen mit allgemeiner Massbestimmung', Jber. Deutsch. Math-Verein 34 (1926), 213-220.

[3] _ _ - 'Untersechung der Krümmung alldemeinen metrischer Räume auf Grund des in ihnen herrschenden Parallelismus', Math. Z. 25 (1926), 40-73.

[4] - 'Parallelubertragung in allgemeinen Räumen', Atti Congr. Intern. Mat. Bologna 4 (1928), 263-270.

[5] __ _ 'Über die $n$-diemensionalen Geometrien konstanter Krümmung, in denen die Geraden die kürzenesten sind', Math. Z. 30 (1929), 449-469.

[6] ___ , Über eine characteristic eigenschaft der allgemeinen Räume konstanter Krümmung mit gradlinigen Extremalen', Monatsh. Math. Phys. 36 (1929), 315-330.

[7] G. Birkhoff and S. MacLane, A survey of modern algebra (Macmillan, New York, 1957).

[8] E. Coddington and N. Levinson, Theory of ordinary differential equations (McGraw-Hill, New York, 1955).

[9] M. Fukui and T. Yamada, 'On projective mappings in Finsler geometry', Tensor (N.S.) 35 (1981), 216-222.

[10] M. Matsumoto, 'Projective changes of Finsler metrics and projectively flat Finsler spaces', Tensor (N.S.) 34 (1980), 303-315.

[11] A. Rapcsak, 'Über die bahntreuen Abblildungen metrisher Räume', Publ. Math. Debrecen 8(1961), 285-290.

[12] Z. Shen, Lectures on Finsler geometry (World Scientific, Singapore, 2001).

[13] J. Stoker, Differential geometry, Pure and Applied Mathematics 20 (Wiley-Interscience, New York, 1969).

[14] Z. I. Szabo, 'Positive definite Berwald spaces (Structure theorems on Berwald spaces)', Tensor (N.S.) 35 (1981), 25-39.

Mathematics

Institute of Fundamental Sciences

Massey University

Private Bag 11222

Palmerston North

New Zealand

e-mail: P.Senerath@massey.ac.nz

G.Thornley@massey.ac.nz

B.vanBrunt@massey.ac.nz 